\title{
Structures and visco-elastic properties of potassium tellurite: glass versus melt
}

\author{
John Kieffer $^{1}$, Jacqueline A Johnson ${ }^{2}$, Oleg Nickolayev ${ }^{3}$ and Jay D Bass ${ }^{4}$ \\ ${ }^{1}$ Department of Materials Science and Engineering, University of Michigan, 2300 Hayward \\ Street, Ann Arbor, MI 48109, USA \\ 2 Energy Technology Division, Argonne National Laboratory, 9700 S Cass Ave, Argonne, \\ IL 60439, USA \\ 3 Department of Materials Science and Engineering, University of Illinois, 1304 West Green \\ Street, Urbana, IL 61801, USA \\ ${ }^{4}$ Department of Geology, University of Illinois, 1301 West Green Street, Urbana, IL 61801, USA
}

Received 17 August 2005, in final form 23 November 2005

Published 6 January 2006

Online at stacks.iop.org/JPhysCM/18/903

\begin{abstract}
The structure and visco-elastic properties of $\mathrm{K}_{2} \mathrm{Te}_{4} \mathrm{O}_{9}$ have been examined as a function of temperature, using neutron scattering and Brillouin light scattering, respectively. The neutron scattering data indicate that the coordination of tellurium by oxygen changes notably once the material is heated above the glass transition temperature. This and the associated decrease in elastic modulus are consistent with converting network building blocks from trigonal bipyramids to trigonal pyramids. The latter form chain-like structures that constitute a liquid characterized by a single relaxation mechanism. Structural relaxation in the liquid results in further decrease of its elastic storage capacity and in a maximum of dissipative losses due to viscous processes. The breakup of the glassy network, which is attributed to a frictionless transformation of building blocks, is distinct from the viscous relaxation of the liquid; their visco-elastic signatures can be observed in separate temperature intervals.
\end{abstract}

(Some figures in this article are in colour only in the electronic version)

\section{Introduction}

Tellurite glasses have received a great deal of attention because of the variety of their potential applications, including in low-loss optical fibres [1-3], as a host material for luminescent rare earth cations and optical amplifiers [4-6], in frequency up-conversion [2, 7, 8], for photo-induced second-order non-linear optical effects [9-11] and in electronically conducting glasses $[12,13]$. Tellurium oxide is an atypical glass former, since by itself it does not form an amorphous solid, except perhaps at extremely high quench rates. The addition of modifier cations, on the other hand, which reduces the glass-forming ability of many networked oxides, stabilizes tellurite glasses. 
The basic structural unit in tellurite glasses is a $\mathrm{TeO}_{4}$ trigonal bipyramid (tbp), where one of the equatorial sites is occupied by a lone pair of electrons [14]. Most of these tbps are connected via oxygen atoms whose linkage to the tellurium atoms is equatorial in one and axial in the other unit. In tbps tellurium is surrounded by four oxygen atoms and all of them are bridging. In terms of nuclear magnetic resonance (NMR) nomenclature, which we find to provide a concise description of the structure in question, this unit is labelled as $Q_{4}^{4}$ [15]. The addition of an alkali oxide modifies this network by creating non-bridging oxygens (NBO). These NBO have a negative charge associated with them, and tbps are deformed into lowersymmetry $\mathrm{TeO}_{3+1}^{-}$polyhedra (or $Q_{4}^{3}$ ). In low-alkali glasses $\mathrm{TeO}_{4}$ tbps and $\mathrm{TeO}_{3+1}$ units form a continuous network. When the amount of alkali is increased to between 20 and $30 \mathrm{~mol} \%$, $\mathrm{TeO}_{3}$ trigonal pyramids (tp) start to appear. This unit has three oxygen atoms connected to tellurium, of which only two are bridging. The NBO forms a double bond with tellurium and hence it is labelled as $Q_{3}^{2}$. While this reduces the functionality of the unit, an entangled network of chain-like structures still prevails. End members of such structures have a singly bonded NBO and can be characterized as $Q_{3}^{1}$. When the alkali content is above $30 \%$, isolated structural units, such as $\left(\mathrm{Te}_{2} \mathrm{O}_{5}\right)^{2-}$, i.e. two $Q_{3}^{1}$ units connected to each other, detach from the network, and at about $50 \%$ alkali oxide $\left(\mathrm{TeO}_{3}\right)^{2-}$ (or $Q_{3}^{0}$ ) monomers are detected along with all the aforementioned entities. In general, the fraction of tbps in the structure decreases with increasing alkali content. The addition of transition metal oxides to $\mathrm{TeO}_{2}$ has similar effects [16] as far as the evolution of network structural units with changes in composition is concerned.

The structural information about tellurite glasses known to date has been obtained from a variety of experimental techniques, including infrared and Raman spectroscopy [16], NMR [17, 18] and EXAFS [19]. Most work focused on the compositional dependence of structural characteristics. The effect of temperature has been little investigated. Tatsumisago et al carried out Raman scattering [20,21] and high-temperature $\mathrm{x}$-ray diffraction measurements [22], both for alkali tellurite glasses and the corresponding melts. Contrasting the data for glasses and melts, the authors concluded that a number of network units shift from tbp to tp upon heating, and that this modification is completely reversible upon cooling. They propose a mechanism by which this can be accomplished without a change in composition, and without violating the condition of electroneutrality.

In the present paper we discuss the structural evolution in potassium tellurite containing $20 \mathrm{~mol} \% \mathrm{~K}_{2} \mathrm{O}$ between room temperature and $900 \mathrm{~K}$. We base our discussion on the results from two techniques of investigation: neutron scattering and Brillouin light scattering. While the former directly gauges the spacing between atomic positions, the latter probes the way in which structural building blocks are connected with one another, and serves as an indirect means of structural characterization. Analysis of Brillouin spectra yields a complex mechanical modulus of structures on a scale of a hundred nanometres. The real component, or storage modulus, reflects the ability of the structure to transmit energy elastically and measures the degree of networking and structural integrity. The loss modulus accounts for viscous dissipation associated with aperiodic movements of structural moieties; it depends on the abundance and mobility of structural defects.

The quantities determined by Brillouin scattering therefore primarily provide insight into the topology of the investigated structure rather than its geometry. In the context of glass formation, however, this measurement has an additional benefit in that it provides a quantitative assessment of the extent of relaxation that a given structure is capable of in thermal equilibrium. The value of Brillouin scattering as a structural probe lies in its ability to very sensitively monitor structural changes as a function of temperature, pressure or chemical composition $[23,24]$. Here we use this information in conjunction with that obtained using 
a more traditional structural characterization technique, i.e. neutron scattering, to elucidate the differences between the structures of molten and glassy potassium tellurite as well as the nature of the transition leading from one structure to the other.

\section{Experimental procedure}

\subsection{Sample preparation}

Glasses were prepared from powders of tellurium oxide and alkali carbonates, with a total impurity content of less than $0.2 \%$ by weight. The powders were intimately mixed, then heated and melted in an open silica crucible and ambient atmosphere. The melt was homogenized at $600{ }^{\circ} \mathrm{C}$ for a period of time sufficiently long to eliminate all gas bubbles from the melt. This happened typically within a matter of 20-30 min. We chose the homogenization time to be as short as possible in order to minimize any dissolution of silica from the crucibles. The chemical composition of the samples was verified after the spectra had been recorded, and showed only trace amounts of impurities.

The sample holders for the light scattering experiment were made of a short piece of fused silica tubing, about $8 \mathrm{~mm}$ long and $4 \mathrm{~mm}$ in diameter, open at both ends. The tube was laterally attached to a silica rod, by which it was suspended from the top cover of the furnace. The sample holders were filled with material by simply immersing them into the molten glass, using capillary forces to hold the liquid in place. For neutron scattering the aforementioned ingredients were melted, homogenized and poured onto a copper plate in several batches to amass a larger quantity. The quenched glass was crushed and about $20 \mathrm{~g}$ was loaded into a silica glass tube of $1 \mathrm{~cm}$ diameter which was sealed at the lower end and connected to a vacuum pump at the upper end. The glass was remelted and held under a low vacuum for several minutes before the tube was sealed at the upper end to form and ampoule about $8 \mathrm{~cm}$ long.

\subsection{Brillouin light scattering}

Details concerning the light scattering experiments can be found in previous publications [23, 24]. Here we summarize the most salient points only. For Brillouin scattering measurements the samples were mounted in a small resistance-heated furnace. The furnace has three orifices — on the top, bottom and side — which were sealed with fused silica windows to maintain a controlled gas atmosphere of air with less than $3 \mathrm{ppm}$ humidity. The samples were first heated to their maximum temperature and spectra were collected upon cooling of the samples at intervals of approximately $20^{\circ} \mathrm{C}$.

The laser beam was brought in from the bottom and the scattered light was collected through the side opening. This way light entered the sample through the open bottom of the sample holder and the scattered light was collected laterally through the cylinder wall, at an average angle of $90^{\circ}$. The incident light was produced by a single-mode argon laser at a wavelength of $0.5145 \mu \mathrm{m}$. The line width of the incident light was approximately $100 \mathrm{MHz}$. Scattered light was collected over a solid angle of about $0.5 \mathrm{sr}$, as determined by the external optics, and analysed using a six-pass tandem Fabry-Perot interferometer with a finesse of about 150 [25], i.e. the width of a perfectly elastic peak would measure less than $1 \%$ of the spectral range of the instrument. The broadening of the Brillouin peaks was caused by the spread in scattering angles due to the finite collection aperture, and was furthermore amplified by the curvature of the silica cylinder. This instrumental broadening was eliminated from the data by means of deconvolution. 
The spectrum of scattered light can be described by a dynamic structure factor, $S(\mathbf{q}, \omega)$, multiplied by a population probability function. For fluids, an expression for the dynamic structure factor can be derived using the generalized hydrodynamic formalism [26] or the mode coupling theory [27]. In the present analysis, we use the expression for $S(\mathbf{q}, \omega)$ resulting from the generalized hydrodynamic approach, which is based on the simultaneous solution of the equations of balance for mass, momentum and energy. Accordingly, the normalized scattering intensity can be expressed in simplified form as

$$
\begin{aligned}
& \frac{S(\mathbf{q}, \omega)}{S(\mathbf{q})}=\frac{(\gamma-1)}{\gamma} \cdot \frac{2 q^{2} \cdot \kappa / \rho_{0} c_{p}}{\omega^{2}+\left(q^{2} \cdot \kappa / \rho_{0} c_{p}\right)^{2}} \\
& \quad+R_{\mathrm{B}}\left(\frac{q^{2} \Gamma_{\mathrm{S}}}{\left(\omega+c_{\mathrm{S}} q\right)^{2}+\left(q^{2} \Gamma_{\mathrm{S}}\right)^{2}}+\frac{q^{2} \Gamma_{\mathrm{S}}}{\left(\omega-c_{\mathrm{S}} q\right)^{2}+\left(q^{2} \Gamma_{\mathrm{S}}\right)^{2}}\right),
\end{aligned}
$$

where $\gamma=c_{p} / c_{v}$, the ratio between the specific heats at constant pressure and constant volume, $\Gamma_{\mathrm{S}} \approx \frac{1}{2}\left[\frac{\eta_{0}}{\rho_{0}}+\frac{c_{\infty}^{2}-c_{0}^{2}}{1+\left(\omega_{\mathrm{S}} \tau_{\mathrm{B}}\right)^{2}} \tau_{\mathrm{B}}\right], \rho_{0}$ is the average density, $\kappa$ is the thermal conductivity, $c_{0}, c_{\infty}$ and $c_{\mathrm{S}}$, are the velocities of sound at zero frequency, at infinite frequency and at the frequency at which the inelastic scattering event occurs $\omega_{\mathrm{S}}=q c_{\mathrm{S}}$, respectively. $q$ is the wavevector of the acoustic phonon that is probed in the experiment and is given by $q=4 \pi n / \lambda \sin \theta / 2$, where $n$ is the refractive index of the medium, $\lambda$ the wavelength of the probing light and $\theta$ the scattering angle. $R_{\mathrm{B}}$ is a factor that depends in a complicated way on $c_{0}, c_{\mathrm{S}}, \omega_{\mathrm{S}}, \gamma, q$ and $\tau_{\mathrm{B}}$ [24], but for all practical purposes is a constant for a given experiment. For amorphous materials Brillouin spectra exhibit up to two doublets of peaks representing the Doppler-shifted light, one resulting from interactions with transverse and the other one with longitudinal sound waves. $\omega_{\mathrm{S}}$ represents the frequency shift of these peaks relative to that of the incident radiation. It is related to the elastic storage modulus according to

$$
M^{\prime}=\frac{\rho_{0} \omega_{\mathrm{S}}^{2}}{q^{2}} .
$$

The full width at half maximum of the Brillouin peaks provides a measure for the sound attenuation, i.e. $\Delta \omega_{\mathrm{B}}=2 q^{2} \Gamma_{\mathrm{S}}$, and from this we can evaluate the loss modulus according to

$$
M^{\prime \prime}=2 \omega_{\mathrm{S}} \rho_{0} \Gamma_{\mathrm{S}} .
$$

The storage and loss modulus constitute the two components of the complex mechanical modulus, which describes the dynamics of the structure under investigation. In the present case the correlation length of the probing deformation is of the order of several hundred interatomic spacings. It is therefore capable of resolving structural features that are of key importance to the glass formation process, such as critical crystallization nuclei. The mechanical actuation of the structure is realized by means of thermal phonons present in the substance at finite temperatures, and therefore the thermodynamic equilibrium of the structure is not compromised in this experiment. Hence, this method is superbly qualified for the investigation of structural developments in supercooled liquids.

\subsection{Neutron scattering}

The neutron diffraction measurements were made at the intense pulsed neutron source (IPNS), Argonne using the glass, liquids and amorphous materials diffractometer (GLAD) [28]. For these experiments the glasses were crushed and loaded into thin-walled vanadium tubes. Time-of-flight diffraction data were collected in groups of detectors at scattering angles ranging from $8^{\circ}$ to $125^{\circ}$, using a wavelength range of $0.3-3.9 \AA$. Instrument background and scattering from the sample container were subtracted from the data; scattering from the 


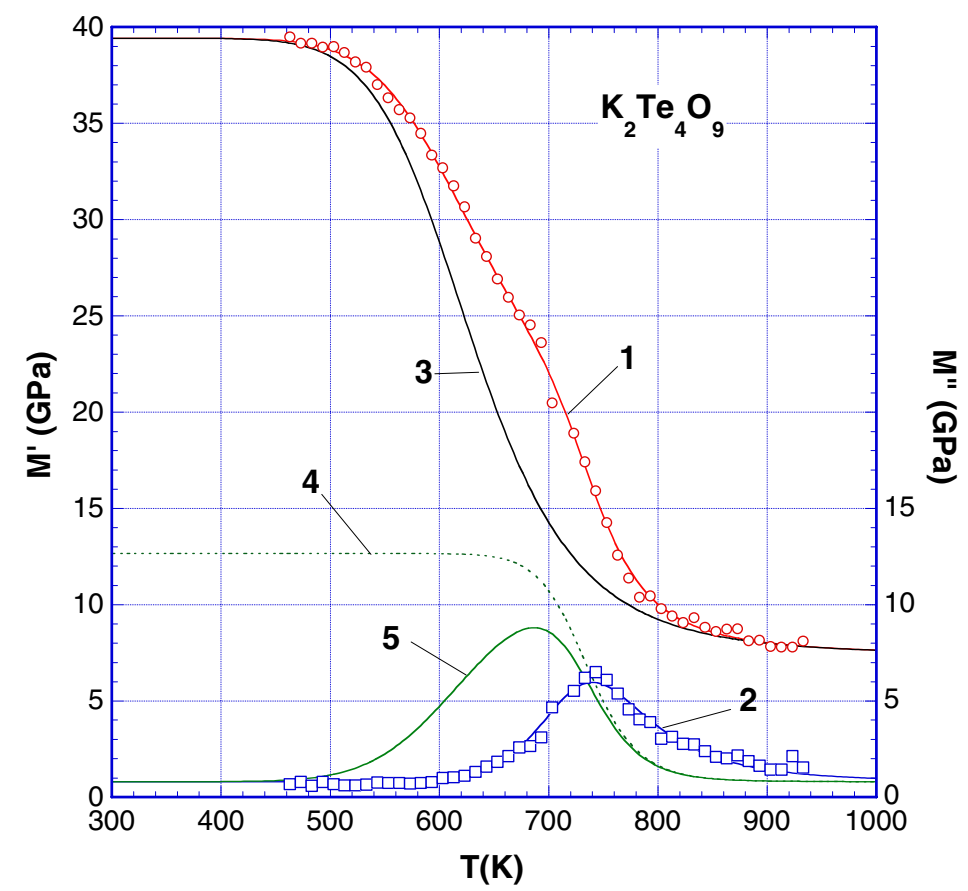

Figure 1. Complex mechanical modulus of the $\mathrm{K}_{2} \mathrm{Te}_{4} \mathrm{O}_{9}$ system as a function of the temperature. Circles represent the measured storage modulus and squares the loss modulus data. Lines represent best fits using the model described with equation (7). The various labels refer to the following parts of this equation: (1) $M^{\prime}$, (2) $M^{\prime \prime}$, (3) $M_{0}+\left(M_{\mathrm{G}}-M_{0}\right) \phi(T)$, (4) $M_{2} \frac{\omega^{2} \tau_{0}^{2} \mathrm{e}^{2 E_{\mathrm{A}} / k_{\mathrm{B}} T}}{1+\omega^{2} \tau_{0}^{2} \mathrm{e}^{2 E_{\mathrm{A}} / k_{\mathrm{B}} T}}$ and (5) $M_{2}(1-\phi(T)) \frac{\omega^{2} \tau_{0}^{2} \mathrm{e}^{2 E_{\mathrm{A}} / k_{\mathrm{B}} T}}{1+\omega^{2} \tau_{0}^{2} \mathrm{e}^{2 E_{\mathrm{A}} / k_{\mathrm{B}} T}}$.

samples was corrected for multiple scattering and absorption and then normalized with respect to the scattering from a vanadium reference [29]. The scattered intensities, $I(Q)$, recorded for the various detector angles and wavelengths were combined to give the neutron weighted average structure factor $S(Q)$ :

$$
I(Q)=|\langle\bar{b}\rangle|^{2}[S(Q)-1]+\left\langle\overline{\left.|b|^{2}\right\rangle}\right.
$$

where the $b$ s are neutron scattering lengths and the angular brackets represent averages over all atoms in the system. Total correlation functions in real space can be defined by Fourier transformation of the corresponding structure factors:

$$
T(r)=4 \pi \rho_{0} r+\frac{2}{\pi} \int_{Q_{\min }}^{Q_{\max }}[S(Q)-1] \sin Q r \cdot Q \mathrm{~d} Q
$$

where $\rho_{0}$ is the total number density. A Lorch modification function was used to reduce termination effects associated with the cut-off at $Q_{\max }$ [30].

\section{Results and discussion}

Figure 1 shows the storage and loss components of the complex mechanical modulus as a function of temperature, measured by Brillouin light scattering. Only longitudinal moduli are shown. These data were obtained during cooling, after heating the sample to its highest 


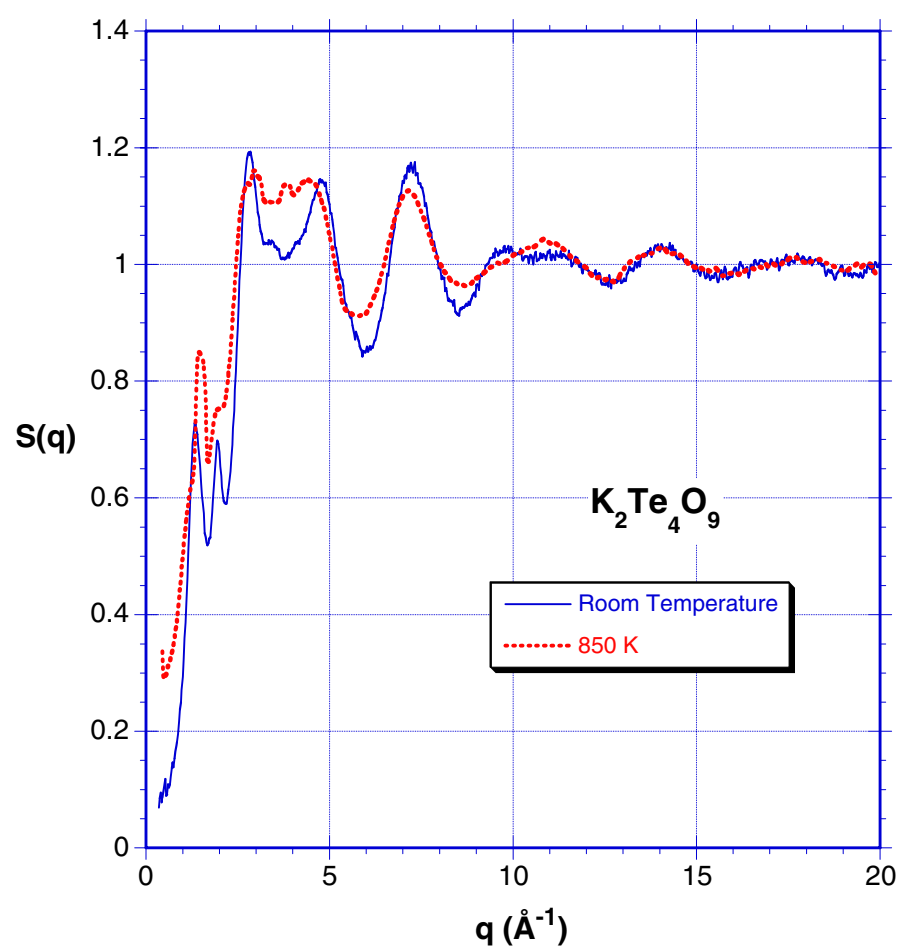

Figure 2. Neutron scattering total structure factors for a $\mathrm{K}_{2} \mathrm{Te}_{4} \mathrm{O}_{9}$ glass at room temperature and melt at $850 \mathrm{~K}$.

temperature. The sample was held at each temperature for about 12-15 min, until thermal equilibrium was reached. The collection of a Brillouin spectrum typically took only 2-3 min. We were able to collect data down to $460 \mathrm{~K}$, which is somewhat below the glass transition temperature of $503 \mathrm{~K}$ for this composition. At this temperature the glass crystallized, which is not entirely surprising given the slow cooling rate. The glass transition is marked by a change in slope in the elastic modulus versus temperature. For this particular composition the change in slope is not very distinct and appears as a gradual bend. Moreover, due to the paucity of data below $T_{\mathrm{g}}$ the exact slope in this temperature regime cannot be discerned with great certainty. The effect is more obvious in other tellurite compositions [24], and even more so in stronger glass-forming systems [23, 31].

In most glass-forming systems we investigated, the temperature dependence of the elastic modulus is weakest below $T_{\mathrm{g}}$, while immediately above the glass transition the elastic storage capacity decays most rapidly with temperature. At high temperatures, well into the liquid regime, the longitudinal modulus gradually levels out due to the finite compressibility of melts. In alkali tellurites, we observe a different behaviour. A second change of slope in the elastic modulus versus temperature data occurs approximately $200 \mathrm{~K}$ above the glass transition. In fact, for tellurites the most rapid decay of elastic modulus takes place only above this second discontinuity.

In figure 2 we compare the neutron scattering structure factors of a room-temperature glass and a melt at $850 \mathrm{~K}$. The latter represents the high-temperature plateau regime in which the liquid can no longer sustain shear deformations, even at GHz-frequency actuation, and the elastic modulus can essentially be attributed to bulk compressive modes of deformation. We observe significant differences between the structure factors of the glass and the liquid. 
(a)

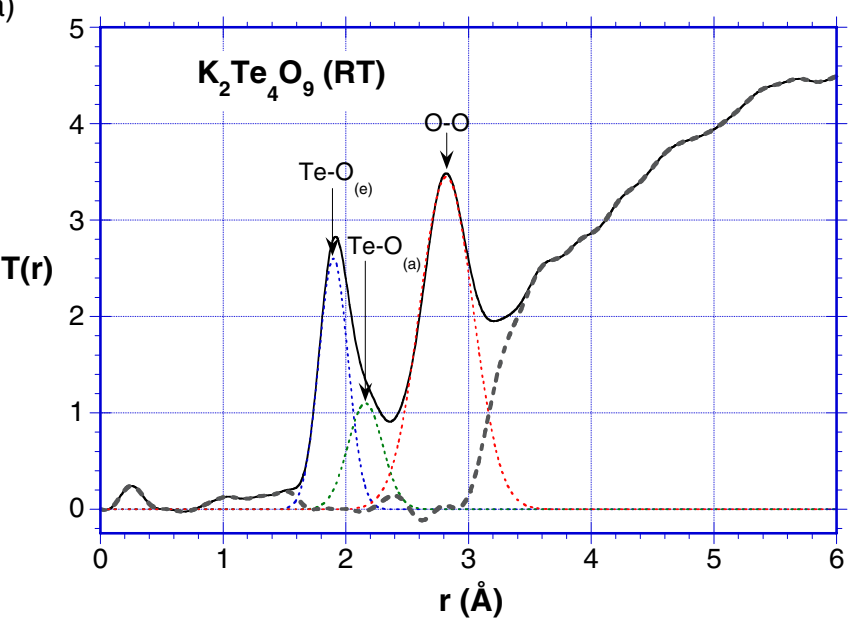

(b)

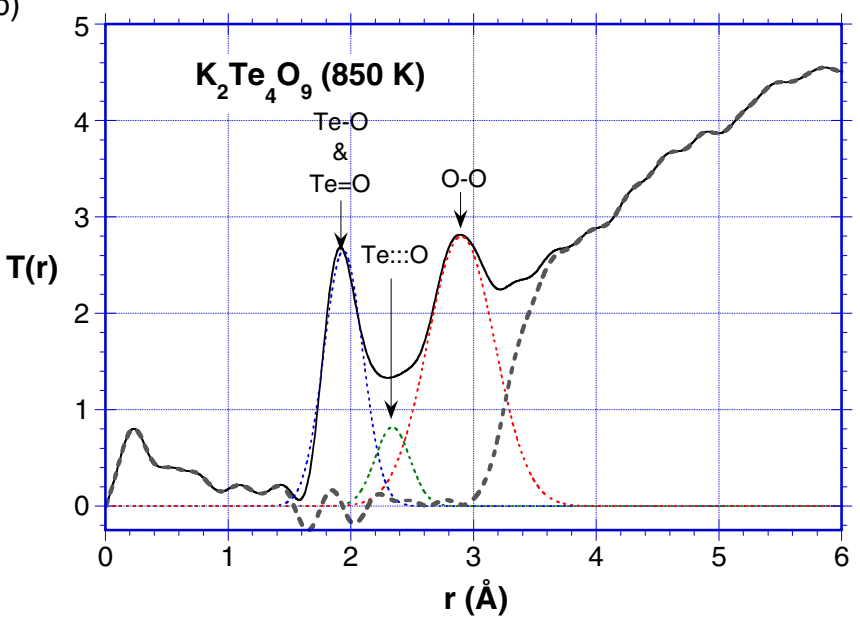

Figure 3. Modified radial distribution functions, $T(r)$, of $\mathrm{K}_{2} \mathrm{Te}_{4} \mathrm{O}_{9}$, (a) at room temperature in the glassy state and (b) at $850 \mathrm{~K}$ in the molten state. Dashed lines are Gaussian fits to $T(r)$, and correspond to the interatomic spacings of tellurium-equatorial oxygen bonds $\left(\mathrm{Te}-\mathrm{O}_{(\mathrm{e})}\right)$, telluriumaxial oxygen bonds $\left(\mathrm{Te}-\mathrm{O}_{(\mathrm{a})}\right)$, tellurium-oxygen single $(\mathrm{Te}-\mathrm{O})$ and double $(\mathrm{Te}=\mathrm{O})$ bonds in tps, broken tellurium-equatorial oxygen bonds $(\mathrm{Te}-\mathrm{O})$ and oxygen-oxygen nearest neighbours $(\mathrm{O}-\mathrm{O})$.

Besides the somewhat stronger damping in the oscillations of the structure factor for the molten sample, some features, especially between 2 and $5 \AA^{-1}$, seem to be entirely missing.

Figure 3 shows the modified radial distribution functions, $T(r)$, of the $\mathrm{K}_{2} \mathrm{Te}_{4} \mathrm{O}_{9}$ glass and melt. These functions are obtained by Fourier transform of the neutron scattering structure factors shown in figure 2. The most distinctive features in these radial distribution functions are the two peaks near 2 and $3 \AA$. The first of these peaks represents the spatial arrangement of Te-O nearest neighbours, and the second peak that of $\mathrm{O}-\mathrm{O}$ nearest neighbours. The dashed lines represent best fits of Gaussians to these peaks. Note that it is necessary to assume at least three peaks in order to achieve a reasonable fit, which indicates that structural units include different types of $\mathrm{Te}-\mathrm{O}$ bonds. In fact, several different bonding configurations are possible. 
In tbp structural units, which are prevalent in a room-temperature glass, the equatorial Te-O bonds are significantly shorter than the axial ones. Literature reports concerning the exact bond lengths differ, which may be due to varying chemical environments, but all accounts place the axial Te-O bond length clearly above $2 \AA$ and that of the equatorial Te-O bonds markedly below $2 \AA$.

Our data for the room-temperature glass shows that the Te-O nearest neighbour peak can be adequately fitted using two Gaussians, one peaking at $1.89 \AA$ and the other one at $2.16 \AA$. These measures are entirely consistent with other literature reports for the equatorial and axial Te-O bond distances, respectively. The introduction of a modifier oxide into the system allows for the creation of NBO and partially disconnected tbp units. It is generally believed that any bridging oxygen acts as an equatorial oxygen for one $\mathrm{TeO}_{4}$ unit and as an axial oxygen for the other one [14]. Similarly, when replacing one bridging oxygen with two $\mathrm{NBO}$, the proportion of equatorial to axial oxygens will not be perturbed, and any changes in the corresponding $\mathrm{Te}-\mathrm{O}$ bond lengths are likely to be too small to be resolved within the thermal noise.

It is possible that the equilibrium of the network modification reaction shifts with temperature, resulting in a larger fraction of $\mathrm{NBO}$ in the molten state, and that this repartitioning can be held accountable for the change in the visco-elastic behaviour. However, in network modification, the chemical constitution of the glass-forming system plays a primary role. It is more likely that the structural integrity of the glassy network is affected by another mechanism when raising the temperature: the changeover from tbp to tp units. In this process one of the axial Te-O bonds is severed, simply as the result of thermal activation. If the open valence of the tellurium that has thus been freed is not occupied by a free oxygen, a double bond may form with one of the equatorial oxygens of this tellurium. This, in turn, consumes the second valence of this equatorial oxygen, which consequently severs its bond to the next $\mathrm{TeO}_{4}$ unit and strips it of an oxygen.

Hence, the rupturing of an axial $\mathrm{Te}-\mathrm{O}$ bond unleashes a chain reaction, which, unless it is buffered by the presence of free oxygen, results in the dissolution of two network links for each tellurium atom; one axial oxygen is lost and one equatorial oxygen is converted into a NBO, but this one is connected through a double bond. In the ensuing tp units the two bonds with bridging oxygens and the double bond with the NBO are of comparable length, all shorter than $2 \AA$. The bond length distribution is approximated with a Gaussian centred at $1.93 \AA$ in figure 2(b). Note that the fit is not perfect and a better result could probably be achieved with a series of two or more closely spaced Gaussians. Resolving the difference in bond lengths between the double- and single-bonded Te-O entities in this manner would be somewhat speculative and not relevant to this discussion. We focus instead on the distinct peak at $2.34 \AA$. This, we believe, reflects the position of a fourth oxygen relative to the tellurium in particular tp, equivalently the type that has been severed from it by breaking the axial Te-O bond. This oxygen is not covalently bonded to this tp, but its position relative to the central tellurium seems to be remarkably steady. This leads us to conclude that a certain degree of attractive interaction exists between non-bonded tps. The broadening of the $\mathrm{O}-\mathrm{O}$ peak and its shift towards a slightly larger distance also corroborates the dissolution of the original tbp unit and the relative stability of the ensuing oxygen configuration, in which for a given unit one oxygen is separated from the tellurium it was bonded to at low temperatures. The change in the electronic structure of the tellurite building blocks with temperature is also evident from the fact that the melts become increasingly more opaque upon heating. Above $950 \mathrm{~K}$ the transmissivity is too weak to allow for a Brillouin scattering measurement.

This model of converting tbps to tps upon heating was originally proposed by Tatsumisago et al [20,21], and our findings are consistent with it. Moreover, our neutron 
and Brillouin scattering data can be interpreted concurrently based on this premise. There is concurrence in two respects: one is that a structural transition takes place between the roomtemperature glass and the melt, and the second is that this transition is essentially devoid of momentum transfer between structural constituents (i.e. all thermal activation is consumed by the change in electronic structure and not by any displacements of atoms relative to one another). To argue these points, we re-examine the complex mechanical modulus data shown in figure 1.

From the point of view that glass is nothing but a supercooled liquid whose characteristic relaxation time exceeds the window of observation by many orders of magnitude, it is convenient to describe the mechanical response of such a system using Maxwell's relationship for a linear visco-elastic medium. However, we have shown that this perspective is an oversimplification [23, 24]. Specifically, in the Maxwell model the loss and storage modulus are related to one another via a Kramers-Kronig transformation. One indication for this condition to be met is if the peak height in the loss modulus amounts to half of the difference between the high- and low-temperature magnitudes of the storage modulus. This is evidently not the case for our data. The loss modulus rises to less than one-fifth of the difference in storage modulus. This observation has been systematic throughout a range of glass-forming systems that we investigated, and we concluded that Maxwell's model must be omitting a fundamental aspect of the glass transition.

Ultimately, we are able to describe the visco-elastic properties of all glass-forming systems we investigated by assuming that the glass is structurally, thermodynamically and mechanically distinct from the melt, and that a gradual transformation between the glassy and the molten state takes place between the glass transition and melting temperatures. The partition between glassy $(R)$ and liquid $(V)$ states is given as a function of temperature by a Boltzmann distribution,

$$
\phi(T)=\frac{n_{R}}{n_{R}+n_{V}}=\left(1+\mathrm{e}^{-\frac{\Delta H\left(T_{\mathrm{c}}-T\right)}{k_{\mathrm{B}} T_{\mathrm{c}} T}}\right)^{-1},
$$

where $\Delta H$ is the difference in enthalpies between glassy and liquid states and $T_{\mathrm{c}}$ is the temperature at which these two states coexist in thermodynamic equilibrium. Assuming furthermore that, to a first approximation, the glassy state is ideally rigid and only the liquid state is visco-elastic, the complex modulus can be expressed as

$M^{*}(\omega, T)=M_{0}+\left(M_{\mathrm{G}}-M_{0}\right) \phi(T)+M_{2}(1-\phi(T)) \frac{\omega^{2} \tau_{0}^{2} \mathrm{e}^{2 E_{\mathrm{A}} / k_{\mathrm{B}} T}+\mathrm{i} \omega \tau_{0} \mathrm{e}^{E_{\mathrm{A}} / k_{\mathrm{B}} T}}{1+\omega^{2} \tau_{0}^{2} \mathrm{e}^{2 E_{\mathrm{A}} / k_{\mathrm{B}} T}}$,

where $M_{0}$ is the static modulus of the melt, $M_{\mathrm{G}}$ that of the glass and $M_{2}$ is the relaxational modulus. $\tau_{0}$ is the fundamental time constant of the relaxation process, and $E_{\mathrm{A}}$ its activation energy. This expression can be trivially expanded to include a distribution of relaxation times and activation energies, but this is in fact not necessary in the present case. The lines in figure 1 show the best fit of this model, assuming a single relaxation mechanism. Curves labelled (1) and (2) represent the overall best fit to the data. Curve (3) exemplifies the transformation from the glassy to the liquid state. The difference between curve (1) and (3) yields curve (5), which represents the relaxational part of the storage modulus and is solely attributed to the liquid state. Because the liquid state emerges gradually with increasing temperature, as measured by the factor $(1-\phi(T))$, the relaxational modulus initially grows accordingly. Eventually it reaches a maximum and decreases because the characteristic relaxation time drops significantly below the period of the probed phonons.

If the liquid state were to persist down to room temperature, the relaxational modulus would be described by the dotted curve (4), which would relate to the loss modulus via Kramers-Kronig transformation. Instead, the storage modulus grows significantly beyond 
this level until the glass transition is reached, and according to our model this increasing capacity to store elastic energy is achieved through structural changes. Specifically, these changes must result in an increased degree of networking. Indeed, this is the case when tps, which can only polymerize into linear chains, convert into tbps that form three-dimensional networks. Obviously, the latter are not only mechanically more rigid but constitute a different structural state, precluding the applicability of Maxwell's model.

The second point of concurrence pertains to the mechanism of this structural transition. The second change in slope that is observed in the elastic modulus of tellurite systems upon heating is due to the drop in the relaxational modulus. It is apparent because the temperature regimes in which the structure of the glass transitions into that of the liquid and where the characteristic relaxation time of the liquid drops below the oscillatory period of the probing motion are sufficiently separated. By the time the molecular motion in the liquid resonates with the probing frequency over $90 \%$ of the glass is converted into liquid. Accordingly, with increasing temperature the glass transforms into a liquid that at first is stiff and only upon further heating begins to flow in response to deformations at the probing frequency. The decrease in the elastic modulus to the left of the second kink can be essentially attributed to structural changes; the drop corresponds to the difference in adiabatic modulus between two different structures.

The important point to notice, however, is that there is no loss modulus peak associated with this initial drop in modulus. This means that the structural reorganization from glass to liquid takes place without collisions between molecular constituents, such as would occur, for example, if there was a change of nearest neighbours. This observation provides support for the mechanism described earlier by which tbps change into tps, i.e. simply by redistributing the electron density upon changing the bonding structure, while involving only minor shifts in atomic positions. This process does not require atoms to move past one another, and therefore dissipates an insignificant amount of energy through atomic collisions. This is especially so if the oxygen that has been severed from a tbp still remains in close proximity to this unit after the bond is broken, at least while temperatures remain below the second kink, where diffusion is small.

\section{Conclusion}

Both structural characterization using neutron scattering and measurement of visco-elastic properties using Brillouin light scattering reveal that $\mathrm{K}_{2} \mathrm{Te}_{4} \mathrm{O}_{9}$ undergoes significant structural changes when heated from the glassy to the liquid state. Specifically, our findings are consistent with the notion that the unit building blocks undergo transition from trigonal bipyramids to trigonal pyramids. The former units are connected via four bridging oxygen atoms to form a glassy network, while the latter can only link via two oxygen atoms and therefore form linear chains. The third oxygen in tps is connected to the tellurium via a double bond. The transformation of structural building blocks is evident in the increased separation of the first two peaks that can be resolved in the radial distribution function derived from neutron scattering structure factors. This reveals the formation of a different type of oxygen in the coordination shell of tellurium, one that results from severing an axial Te-O bond. The breaking of this bond is frictionless, i.e. it occurs without neighbour exchange and the ensuing interatomic collisions. Furthermore, to maintain electronic balance about tellurium, these bond breaks occur in pairs for each unit. As a consequence, the glass network breaks down precipitously, bringing about a highly viscous melt of chain-like molecules. However, only at higher temperatures does this melt structure relax within the period of phonons probed during the Brillouin experiment. Consequently, the transformation of structural building blocks and 
the viscous relaxation of the melt occur within separate temperature ranges, which ultimately allowed us to distinguish between the two processes.

\section{Acknowledgments}

This work was supported by the National Science Foundation under grant no DMR-0072258, and by the US Department of Energy, Office of Science, under contract no W-31-109-ENG38 .

\section{References}

[1] Durga D K, Reddy P Y and Veeraiah N 2002 Optical absorption and thermoluminescence properties of $\mathrm{ZnF}_{2}-\mathrm{MO}-\mathrm{TeO}_{2}\left(\mathrm{MO}=\mathrm{As}_{2} \mathrm{O}_{3}, \mathrm{Bi}_{2} \mathrm{O}_{3}\right.$ and $\left.\mathrm{P}_{2} \mathrm{O}_{5}\right)$ glasses doped with chromium ions J. Lumin. 99 53-60

[2] Lin $\mathrm{H}$ et al 2003 Optical transitions and visible upconversion in $\mathrm{Er}^{3+}$ doped niobic tellurite glass J. Appl. Phys. 93 186-91

[3] Wallenberger F T, Weston N E and Brown S D 1992 Infrared optical tellurite glass-fibers J. Non-Cryst. Solids 144 107-10

[4] Mori A et al $20021.58 \mu \mathrm{m}$ broad-band erbium-doped tellurite fiber amplifier J. Lightwave Technol. 20 794-9

[5] Shen S X et al 2002 Tellurite glasses for broadband amplifiers and integrated optics J. Am. Ceram. Soc. 85 $1391-5$

[6] Shen S X, Naftaly M and Jha A 2002 Tungsten-tellurite-a host glass for broadband EDFA Opt. Commun. 205 101-5

[7] Poirier G et al 2003 Optical properties and frequency upconversion fluorescence in a $\mathrm{Tm}^{3+}$-doped alkali niobium tellurite glass J. Appl. Phys. 93 3259-63

[8] Rakov N et al 2002 Blue upconversion enhancement by a factor of 200 in $\mathrm{Tm}^{3+}$-doped tellurite glass by codoping with $\mathrm{Nd}^{3+}$ ions J. Appl. Phys. $926337-9$

[9] Qiu J R, Si J H and Hirao K 2001 Photoinduced stable second-harmonic generation in chalcogenide glasses Opt. Lett. 26 914-6

[10] Yonesaki Y, Tanaka K, Si J H and Hirao K 2002 Second-harmonic generation in optically poled tellurite glasses doped with $\mathrm{V}_{2} \mathrm{O}_{5}$ J. Phys.: Condens. Matter 14 13493-503

[11] Yonesaki Y, Tanaka K, Narazaki A, Si J H and Hirao K 2002 Relaxation phenomena in second-order nonlinearity of thermally and optically poled $\mathrm{Nb}_{2} \mathrm{O}_{5}-\mathrm{TeO}_{2}$ glasses J. Phys. D: Appl. Phys. 35 2026-31

[12] Moawad H M M, Jain H, El-Mallawany R, Ramadan T and El-Sharbiny M 2002 Electrical conductivity of silver vanadium tellurite glasses J. Am. Ceram. Soc. 85 2655-9

[13] Rozier P, Dubois T and Salles P 2002 Electronic conductivity and structural chemistry in Li-Te- $\mathrm{V}^{5+}, \mathrm{V}^{4+}$ oxide glasses J. Non-Cryst. Solids $311241-9$

[14] Sekiya T, Mochida N, Ohtsuka A, Tonokawa M and Mamoru J 1992 Raman spectra of $\mathrm{MO}_{1 / 2}-\mathrm{TeO}_{2}$ ( $\mathrm{M}=\mathrm{Li}, \mathrm{Na}, \mathrm{K}, \mathrm{Rb}, \mathrm{Cs}$, and Tl) glasses J. Non-Cryst. Solids 144128

[15] McLaughlin J C, Tagg S L, Zwanziger J W, Haeffner D R and Shastri S D 2000 The structure of tellurite glass: a combined NMR, neutron diffraction, and X-ray diffraction study J. Non-Cryst. Solids 274 1-8

[16] Burger H H, Kneipp K, Hobert H and Vogel W 1992 J. Non-Cryst. Solids 151134

[17] Tagg S L, Youngman R E and Zwanziger J W 1995 J. Phys. Chem. 995111

[18] Youngman R E and Aitken B G 2001 NMR studies of aluminum speciation in tellurite glasses J. Non-Cryst. Solids $2849-15$

[19] Osaka A, Juanrong Q, Nanba T, Takada J and Miura Y 1992 J. Non-Cryst. Solids 14281

[20] Tatsumisago M, Kato S, Minami T and Kowada Y 1995 High-temperature structure and crystallization kinetics of $\mathrm{Li}_{2} \mathrm{O}-\mathrm{TeO}_{2}$ glasses J. Non-Cryst. Solids 193 478-81

[21] Tatsumisago M, Lee S K, Minami T and Kowada Y 1994 Raman-spectra of $\mathrm{TeO}_{2}$-based glasses and glassy liquids-local-structure change with temperature in relation to fragility of liquid J. Non-Cryst. Solids 177 154-63

[22] Akagi R et al 1999 High-temperature structure of $\mathrm{K}_{2} \mathrm{O}-\mathrm{TeO}_{2}$ glasses J. Non-Cryst. Solids 257 111-8

[23] Masnik J E, Kieffer J and Bass J D 1995 The complex mechanical modulus as a structural probe: case of alkali borate liquids and glasses J. Chem. Phys. 103 9907-17

[24] Kieffer J, Masnik J E, Nickolayev O and Bass J D 1998 Brillouin scattering investigation of alkali tellurite melts Phys. Rev. B 58 694-705

[25] Sandercock J R 1982 Topics in Applied Physics vol 51, ed M Cardona and G Güntherodt (Berlin: Springer) pp 173-206 
[26] Mountain R D 1966 J. Res. NBS A 70207

[27] Tao N J, Li G and Cummins H Z 1991 Phys. Rev. B 435815

[28] http://www.pns.anl.gov/instruments/glad/subglad/ 2005

[29] Johnson J A et al 1999 J. Non-Cryst. Solids 246104

[30] Newport R J 1988 Neutron Scattering at a Pulsed Source (Bristol: Hilger)

[31] Masnik J E, Kieffer J and Bass J D 1993 Structural relaxations in alkali-silicate systems by brillouin light scattering J. Am. Ceram. Soc. 763073 